\title{
HALLAZGO DE Dactyloidites ottoi (GEINITZ, 1849) EN LA ARENISCA DE ARÉN (CRETÁCICO SUPERIOR SURPIRENAICO)
}

\author{
Nieves LÓPEZ-MARTÍNEZ' y Luis ARDÉVOL² \\ 'Dep. Paleontología, Fac. C. Geológicas, Universidad Complutense, 28040 \\ Madrid. \\ 'GEOPREP, Nerets 10, 25620 Tremp, Lleida.
}

\begin{abstract}
López Martínez, N. y Ardévol, L. 1999. Hallazgo de Dactyloidites ottoi (Geinitz, 1849) en la Arenisca de Arén (Cretácico Superior surpirenaico). [The finding of Dactyloidites ottoi (Geinitz, 1849) in the Arén Sandstone (Upper Cretaceous, southern Pyrenees)]. Revista Española de Paleontología, $\mathbf{n}^{\circ}$ extr. Homenaje al Prof. J. Truyols, 129-133. ISSN 0213-6937.
\end{abstract}

\begin{abstract}
Trace fossils attributed to Dactyloidites ottoi (Geinitz, 1849) have been found in estuarine, tide-influenced deposits of the Upper Cretaceous Arén Sandstone (South-Central Pyrenees, Spain). The rosette-shaped traces have 12-20 straight or asymmetrically branched radial elements, with a constant width of about $5 \mathrm{~mm}$, covering around 200 degrees of a circle. This ichnotaxon, which was produced by an unknown sedimentivorous animal, has also been found in similar facies in Upper Cretaceous sediments from Germany, Japan and Greenland, and Neogene sandstones from Borneo, Spain and Jamaica. The findings point to a tracemaker living in a lower estuarine environment with a tropical climate.
\end{abstract}

Keywords: Trace fossils, Upper Cretaceous, tidal environment, Pyrenees.

\section{RESUMEN}

En el Cretácico Superior de la Arenisca de Arén (cuenca surpirenaica) se registran pistas fósiles atribuidas a Dactyloidites ottoi (Geinitz, 1849) en sedimentos marinos de estuario con influencia de mareas. Las pistas en forma de roseta tienen 12-20 radios rectos o asimétricamente ramificados, con una sección constante de unos 5 $\mathrm{mm}$, que cubren unos $200^{\circ}$ de círculo. Este icnotaxón, producido por un animal sedimentívoro desconocido, ha sido también encontrado en facies similares en sedimentos del Cretácico Superior en Alemania, Japón y Groenlandia, y en areniscas del Neógeno de Borneo, España y Jamaica. Los hallazgos apuntan hacia un organismo productor habitante de medios de estuario inferior en climas tropicales.

Palabras clave: Icnología, Cretácico Superior, sedimentos mareales, Pirineos.

\section{INTRODUCCIÓN}

La Arenisca de Arén es una formación litoestratigráfica definida por Mey et al. (1968) en el Cretácico Superior de la región sur-central de los Pirineos. Está formada principalmente por areniscas bioclásticas ricas en cuarzo, depositadas en un ambiente marino somero (estuarios, playas e islas barrera), aunque también contiene depósitos de plataforma, sedimentos gravitacionales y turbiditas en los que las areniscas se intercalan con potentes niveles de margas (Ghibaudo et al., 1974; Nagtegaal et al., 1983; Díaz-Molina, 1987). Su interés como modelo de almacén de petróleo ha atraído desde los años sesenta la atención de numerosos expertos.
En este trabajo se describe un tipo de pistas fósile observadas en la Arenisca de Arén en dos localidades Isona (Lleida) y Benabarre (Huesca). Su forma de roset: radiada y su pauta de ramificación es característica d Dactyloidites ottoi (Geinitz, 1849), originalment interpretado como restos fósiles de esponjas Posteriormente, su interpretación ha sufrido numerosa variaciones, y actualmente se consideran como pistas do alimentación de invertebrados (fodinichnia). Presenta un distribución de facies restringida y una ampli distribución espacial y temporal. Pistas similares han sid citadas anteriormente en depósitos de plataform siliciclástica de Groenlandia, Alemania, Japón, Borneo Jamaica y España, desde el Cretácico Superior a 


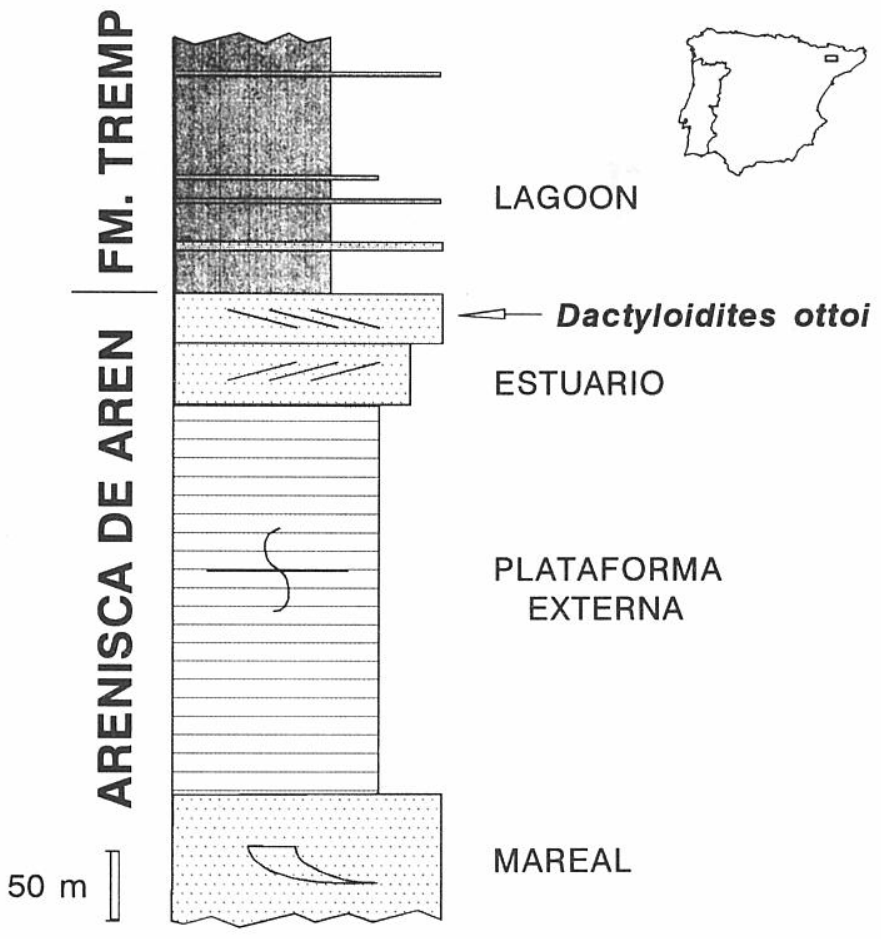

Figura 1. Columna estratigráfica de situación de las pistas. En color claro, margas grises. En color más oscuro, areniscas con estratificación cruzada sigmoidal bimodal, y alternancia de areniscas y margas bioturbadas.

Plioceno. Los hallazgos de épocas más recientes indican que el organismo desconocido que las produjo vivía en climas tropicales.

\section{SITUACIÓN GEOLÓGICA}

Los afloramientos de la Arenisca de Arén forman actualmente un frente relativamente continuo con orientación Este-Oeste, a lo largo de unos 90 km entre los ríos Segre y Ésera, dividido a ambos flancos del sinclinal de Tremp. La potencia llega a sobrepasar $1.000 \mathrm{~m}$. En Benabarre, la Formación Arén, parcialmente cubierta en su base, presenta un desarrollo mínimo de $300 \mathrm{~m}$.

La Arenisca de Arén se relaciona lateralmente y hacia muro con margas y turbiditas de la Formación Vallcarga en dirección Norte y Noroeste (la de la cuenca). Hacia el Este y el Sur (hacia el interior del continente) pasa lateralmente y hacia techo a las capas rojas de la Formación Tremp (Garrido Mejías y Ríos Aragüés, 1972; Ghibaudo et al., 1974; Nagtegaal et al., 1983; DíazMolina, 1987), y a las facies de plataforma de las Calizas de les Serres (López Martínez et al., 1998). Su edad abarca desde el Campaniense superior al Maastrichtiense.

Uno de los afloramientos de Arenisca de Arén que ha proporcionado los fósiles aquí descritos se encuentra en la cuenca de Tremp, en el cierre periclinal del anticlinal de Isona (Lleida). La potencia de la formación Arén sensu lato en el anticlinal de Isona supera ampliamente los $1.000 \mathrm{~m}$ de sedimentos margosos y arenosos. En su techo se ha diferenciado la Formación Arén sensu stricto (Nagtegaal et al., 1983; Willems, 1985), que presenta unos $100 \mathrm{~m}$ de depósitos detríticos organizados en una secuencia grano- y estratocreciente de areniscas ricas en cuarzo con estratificación cruzada sigmoidal bimodal.

Estos depósitos mareales han sido interpretados por Nagtegaal et al., (1983) como producto de un sistema deltaico dominado por las mareas (estuario); en su parte inferior, el sistema prograda en dirección NO (dominarían las corrientes de reflujo), y en su parte superior se invierte la tendencia, dominando las corrientes de flujo. Esta transgresión produciría un tidal inlet retrogradante que invadiría una laguna costera (lagoon), representada por los sedimentos suprayacentes de margas oscuras con lignitos de la Formación Tremp. Esta reconstrucción se basa en la ley de Walter, que interpreta una secuencia vertical como medios relacionados en sentido horizontal, pero aún no ha sido corroborada con correlaciones laterales.

La edad del techo de la Formación Arén s.s. en Isona se ha situado aproximadamente en el Maastrichtiense, por la presencia de rudistas como Radiolitella pulchellus (Vidal) e Hippuritella castroi (Vidal) y macroforaminíferos como Siderolites calcitrapoides y Lepidorbitoides minor (Liebau, 1973; Nagtegaal et al., 1983; Willems, 1985).

La figura 1 muestra la situación de las pistas en la columna estratigráfica de la Formación Arén en Isona. Las pistas fósiles observadas se sitúan en el Barranco de La Posa cerca del techo de la formación. En la figura 2 se observan las areniscas con estratificación cruzada sigmoidal de donde proceden los fósiles.

Se han encontrado también ejemplares de este tipo de pistas en la Arenisca de Arén en Benabarre (Huesca), en facies deltaicas de alta energía hidrodinámica similares a las de Isona. Los afloramientos del Cretácico Superior en esta localidad se encuentran rodeados por conglomerados del Oligoceno pertenecientes a la cuenca del Ebro.

\section{DESCRIPCIÓN DE LAS PISTAS}

Las pistas se observan en bloques ex situ de arenisca de grano medio, rica en granos de cuarzo y en restos vegetales carbonosos. Tienen forma de rosetas radiadas de color más oscuro que la matriz, estando cada radio rodeado de una aureola más clara. La diferencia de color indica una mayor concentración de materia orgánica en el interior de las pistas, aunque no se observan diferencias de textura del material dentro y fuera de la pista. Las rosetas aparecen individualizadas, muy numerosas y próximas entre sí formando agrupaciones dispuestas en planos aproximadamente paralelos al plano de estratificación. En superficie no se observa apenas solapamiento entre ellas. Cubren una amplia área en las zonas donde son visibles, causando una importante bioturbación.

Cada roseta es un círculo formado por pistas radiales, rectas o con pocas ramificaciones, que cubren un área de 200 a $300^{\circ}$. Se observan rosetas que poseen entre 12 y 20 
radios, dependiendo del área de círculo cubierta. Tanto los radios como las rosetas muestran gran regularidad de tamaño y forma. El diámetro de cada roseta mide unos $10-12 \mathrm{~cm}$, y cada radio mide de 4 a $6 \mathrm{~cm}$ de largo.

El grosor de los radios es bastante regular, alrededor de $0,5 \mathrm{~cm}$. Cada radio está rodeado por un halo o espacio más o menos amplio de sedimento más claro que la matriz. Tienen un recorrido recto o ligeramente sinuoso y terminaciones romas. La mayoría presenta ramificaciones que se producen en número máximo de tres, generalmente hacia un solo lado. Los elementos radiales de una roseta pueden ramificarse de forma simétrica, la mitad hacia un lado y la otra mitad hacia el lado contrario.

Tanto los radios rectos como las ramificaciones terminan aproximadamente equidistantes unos de otros en el borde de la roseta, y su longitud aproximadamente constante produce rosetas circulares. En la figura 3 se observa una imagen de estos fósiles, conservados como relieve completo en el interior de las capas de arenisca.

\section{INTERPRETACIÓN Y DISCUSIÓN}

El tamaño y la forma de estas pistas permiten identificarlas como la icnoespecie Dactyloidites otto $i$ (Geinitz, 1849). Este icnofósil fue inicialmente descrito como una esponja, y después atribuido a restos de medusas o de plantas. Häntzschel (1930) reconoció su naturaleza icnológica, y lo consideró moldes de impresiones (hiporrelieves convexos) de invertebrados marinos. Fürsich y Bromley (1985) describen con este nombre unas pistas procedentes del Campaniense de Groenlandia con idéntica forma y tamaño a las aquí descritas y producidas en una litofacies semejante, y las consideran estructuras radiales de galerías de alimentación, con conexiones verticales y relleno en menisco (relieves completos), excavadas por un gusano sedimentívoro. La única diferencia respecto a las pistas de la Formación Arén radica en su frecuente conservación como epirrelieves convexos debido a la erosión.

Los autores asumen que una galería vertical o fuste, orientada oblicuamente al plano de estratificación, conectaría las galerías radiales de cada roseta así como a varias rosetas superpuestas. Las galerías radiales serían pistas de reptación intraestratales que minarían el sedimento. Cada galería tendría una fina envuelta de partículas ricas en restos vegetales que se erosionaría, de modo que originalmente los radios habrían estado en estrecho contacto entre sí aprovechando todo el espacio.

Según los autores, cada radio de la roseta estaría formado, primero por la excavación de una galería horizontal progradante; luego por el desplazamiento uniforme hacia abajo de la galería simultáneamente en toda su longitud (conexiones sucesivas); y posteriormente por el abandono de la galería final por el organismo y su relleno en menisco. La sección transversal de un radio presenta una clara diferenciación, más clara hacia techo (zona de conexiones) y más oscura hacia el muro (relleno de la galería final, más rico en materia orgánica).

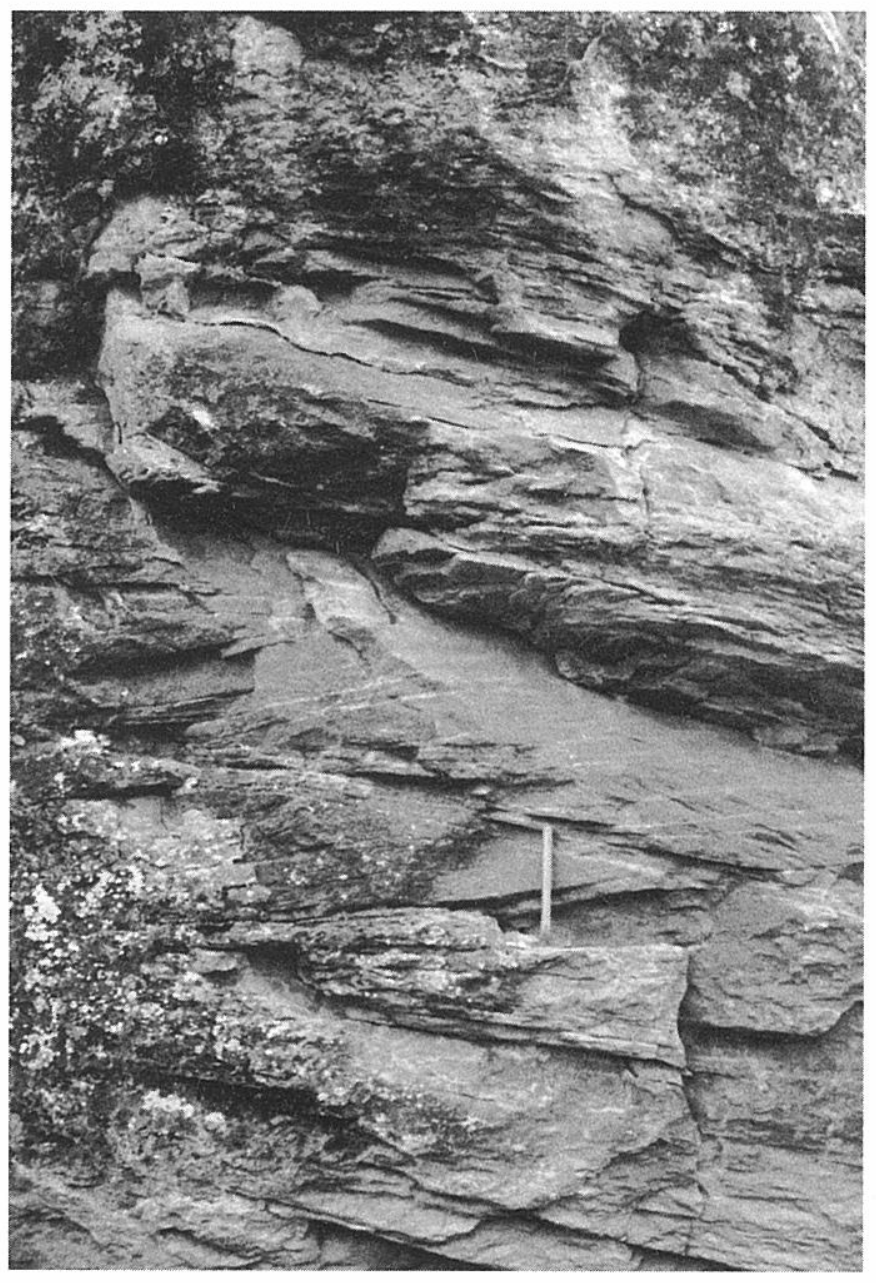

Figura 2. Techo de la Arenisca de Arén en Isona (Lleida, Cretácico Superior) de donde proceden las pistas fósiles. Se observa estratificación cruzada sigmoidal indicando la acción de las mareas; los foresets están orientados al SE (hacia el continente), y han sido interpretados como producto de un tidal inlet (dominaría la corriente de flujo).

Esta icnoespecie ha sido citada siempre en sedimentos arenosos costeros con estratificación cruzada, en el Cretácico Superior de Alemania (Häntzschel, 1930), ?Japon (Tanaka y Sumi, 1981) y Groenlandia (Fürsich y Bromley, 1985), en el Mioceno de Tarragona (Gibert et al., 1995) y en el Plioceno de Jamaica (Pickerill et al., 1993). Estos últimos autores consideran también Haentzschelinia labuanensis Keij 1965, del Mioceno de Borneo, sinónimo de Dactyloidites ottoi.

En el caso de Dactyloidites ottoi de la Arenisca de Arén no se ha observado ninguna galería vertical o fuste en el centro de las rosetas, aunque en otros casos esta galería se observa raramente (Pickerill et al., 1993; Gibert et al., 1995) o no se observa (Tanaka y Sumi, 1981). El número de elementos radiales de cada roseta, así como el sector de círculo que cubren parece ser mayor en los ejemplares descritos de sedimentos cretácicos $\left(12\right.$ a $\left.25,200-300^{\circ}\right)$ que en los neógenos (5$\left.13,120-150^{\circ}\right)$. También en los casos más recientes la 


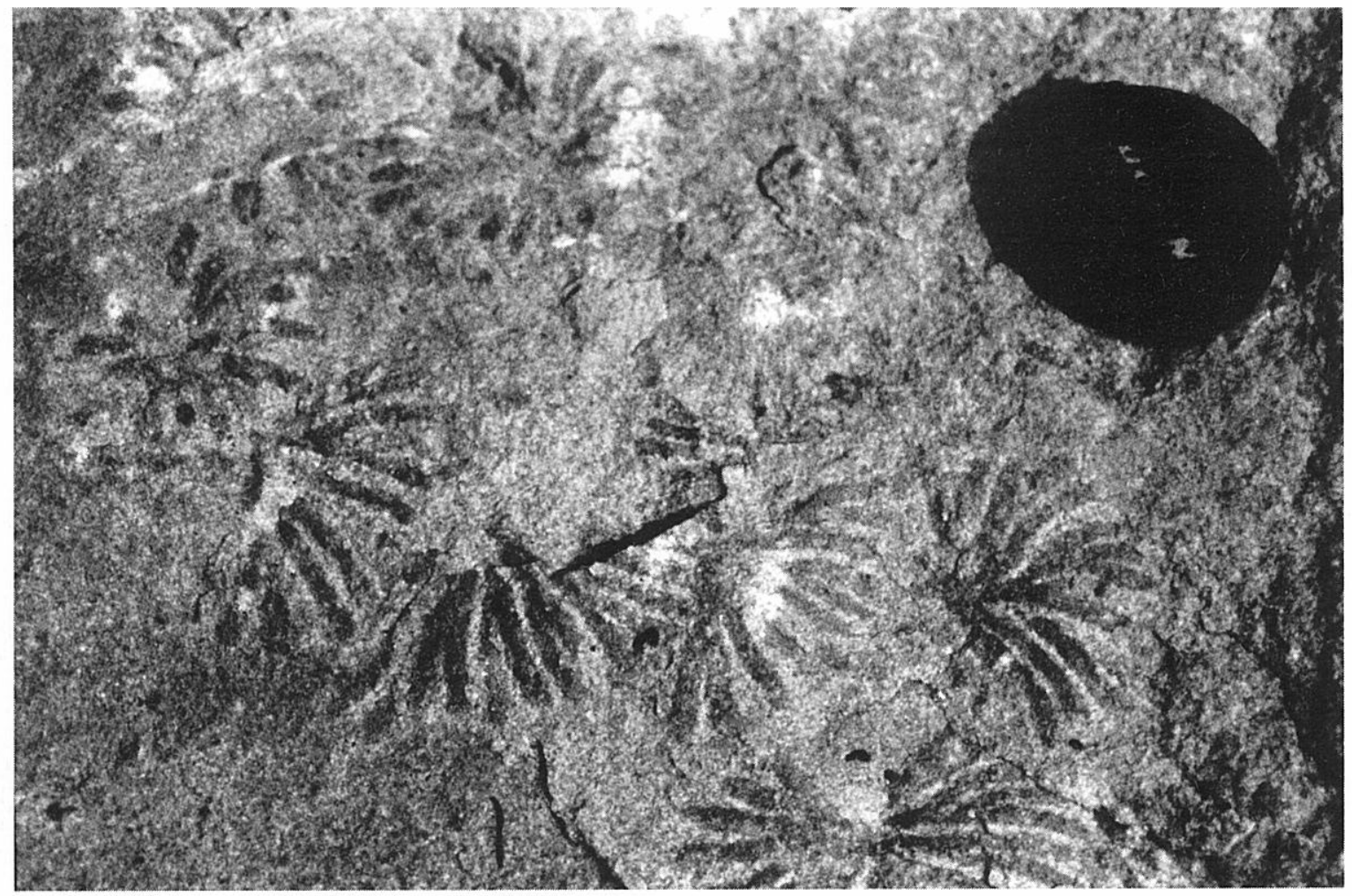

Figura 3. Pistas fósiles identificadas como Dactyloidites ottoi (Geinitz) en el techo de la Arenisca de Arén, (Cretácico Superior de Isona, Lleida). Cliché, I. Munuera. Diámetro del objeto de escala, aproximadamente $5 \mathrm{~cm}$.

longitud y grosor de los elementos radiales y su espaciado es más irregular.

No se conoce el organismo productor, que según los autores citados tendría gran movilidad. Suponen que sería un organismo vermiforme, quizá dotado de una probóscide, que se extendería a distancia constante desde el eje de la roseta donde permanecería una parte fija del animal. Un mismo organismo podría producir todas las rosetas de un grupo o racimo, y perforar una galería de escape cuando el sedimento no fuera adecuado. Se le atribuye un comportamiento complejo con una pauta de intensa explotación del sedimento. Las rosetas exactamente superpuestas permiten explotar el sedimento completamente en dirección vertical, y gracias a abarcar sólo dos tercios de círculo, las rosetas adyacentes pueden cubrir el espacio en la horizontal casi en su totalidad.

Vyalov (1964) describe en el Triásico de Siberia varias icnoespecies del icnogénero Haentzschelinia que Fürsich y Bromley (1985) consideran tentativamente como posibles sinónimos de Dactyloidites ottoi. Otras icnoespecies atribuidas por estos autores a este icnogénero tienen sin embargo pautas de formación muy diferentes, aunque la forma final presenta ciertas semejanzas. Así, la especie tipo D. asterioides (Fitch) del Cámbrico Inferior, posee pocos radios rectos cubriendo un círculo completo, pero no están formados por galerías horizontales sino por conexiones sucesivas de una galería recurvada en forma de J. También $D$. canyonensis (Bassler) difiere de Dactyloidites ottoi por poseer escasos radios rectos que forman un círculo completo, y conexiones que muestran un movimiento ascendente en vez de descendente. Según Pickerill et al. (1993), D. peniculus D'Alessandro y Bromley es una icnoespecie diferente de $D$. ottoi por tener muy numerosos elementos no ramificados y retrogradantes.

Dactyloidites ottoi representa un tipo especial de pistas fósiles ligadas a facies detríticas costeras de tipo estuarino y particularmente extendidas en el hemisferio Norte durante el Cretácico Superior. Gibert et al. (1995) consideran probablemente significativa su presencia durante el Neógeno en áreas con clima tropical. Esta indicación puede efectivamente ser retenida, ya que muchas fuentes de evidencia permiten inferir que durante el Cretácico Superior gran parte del actual Hemisferio Norte debía encontrarse bajo este tipo de clima.

\section{AGRADECIMIENTOS}

Queremos desde estas páginas reconocer con admiración la trayectoria del Dr. Jaime Truyols, Catedrático de Paleontología de la Universidad de Oviedo; su trato humano, su amplitud de observación y su gran capacidad de estudio, desde fósiles del Paleozoico al Terciario y desde invertebrados a mamíferos son un ejemplo para todos. Agradecemos la ayuda de Rodrigo Soler Gijón, Pep Capdevila e Isabel Munuera en distintas fases de este trabajo. El proyecto PB95-0368 de la DGICYT proporcionó soporte económico. Los editores y revisores de la Revista Española de Paleontología han contribuido notablemente a mejorar el manuscrito original, lo que agradecemos sinceramente. 


\section{BIBLIOGRAFÍA}

Díaz-Molina, M. 1987. Sedimentación sintectónica asociada a una subida relativa del nivel del mar durante el Cretácico superior (Fm. Tremp, provincia de Lérida). In: Geología y Paleontología (Arcosaurios) de los yacimientos de Galve (Teruel, Cretácico inferior) y Tremp (Lérida, Cretácico superior) (Ed., J.L. Sanz). Estudios Geológicos, Volumen extraordinario, Galve-Tremp, 69-93.

Fürsich, F.T. and Bromley, R.G. 1985. Behavioural interpretation of a rosetted spreite trace fossil: Dactyloidites ottoi (Geinitz). Lethaia, 18, 199-207.

Garrido Megías, A. y Ríos Aragües, L.M. 1972. Síntesis geológica del Secundario y Terciario entre los ríos Cinca y Segre. Boletín Geológico y Minero, 83, 1-47.

Geinitz, H. B. 1849. Das quadersandsteingebirge oder Kreidegebirge in Deutschland. Craz und Gerlach, Freiberg, $292 \mathrm{pp}$.

Ghibaudo, G., Mutti, E. e Rosell, J. 1974. Le spiagge fossili delle Arenarie de Arén (Cretacico Superiore) nella valle Noguera Ribagorzana (Pirinei centro-meridionali, provincia de Lérida e Huesca, Spagna). Memoria della Società Geologica Italiana, 13, 497-537.

Gibert, J. M. de, Martinell, J. and Domènech, R. 1995. The rosetted feeding trace fossil Dactyloidites ottoi (Geinitz) from the Miocene of Catalonia. Geobios, 28, 769-776.

Häntzschel, W. 1930. Spongia ottoi Geinitz, ein sternförmiges Problematikum aus dem sächsischen Cenoman. Seckenbergiana, 12, 261-274.

Keij, A.J. 1965. Miocene trace fossils from Borneo. Paläontologische Zeitschrift, 39, 220-227.
Liebau, A. 1973. El Maastrichtiense lagunar ("Garumniense”) de Isona. XIII Coloquio Europeo Micropaleontología (España), ENADIMSA, Madrid, 87-112.

López-Martínez, N., Ardévol, L., Arribas, M.E., Civis, J. and González-Delgado, J.A. 1998. The geological record in continental environments around the K/T boundary (Tremp Formation, Spain). Bulletin de la Societé géologique de France, 169, 11-20.

Mey, P., Nagtegaal, P., Roberti, K. and Hartevelt, J. 1968. Lithostratigraphic subdivision of posthercynian deposits in the South-Central Pyrenees, Spain. Leidse Geologische Mededelingen, 41, 221-228.

Nagtegaal, P. J. C., Van Vliet, A. and Brouwer, J. 1983. Syntectonic coastal offlap and concurrent turbidite deposition: the Upper Cretaceous Aren Sandstone in the South Central Pyrenees, Spain. Sedimentary Geology, 34, 185-218.

Pickerill, R. K., Donovan, S. K. and Dixon, H. L. 1993. The trace fossil Dactyloidites ottoi (Geinitz, 1849) from the Neogene August Town Formation of South Central Jamaica. Journal of Paleontology, 67, 1070-1074.

Tanaka, K. and Sumi, Y. 1981. Cretaceous paleocurrents in the central zone of Hokkaido, Japan. Bulletin Geological Survey of Japan, 32, 65-128.

Vyalov, O.S. 1964. Zvezdchatye ierogliphy iz Triasa severovostoka Sibiri. Akademia Nauk SSSR, Sibirsk Otdel Geologii Geofizike, 112-115.

Willems, H. 1985. Marine uppermost Cretaceous and Garumnian facies in the region of Boixols-Coll de Nargo anticline (prov. Lerida, Spain). Estudios geológicos, 41, 17-24.

Manuscrito recibido: 15 de enero, 1998 Manuscrito aceptado: 31 de mayo, 1998 